\title{
Pilot-Based Adaptive Channel Estimation for Underwater Spatial Modulation Technologies
}

\author{
Naveed Ur Rehman Junejo ${ }^{1}$, Hamada Esmaiel ${ }^{2}{ }^{\mathbb{D}}$, Haixin Sun ${ }^{1, * \mathbb{D}}$, Zeyad A. H. Qasem ${ }^{1}$ \\ and Junfeng Wang ${ }^{3}$ \\ 1 Key Laboratory of Underwater Acoustic Communication and Marine Information Technology, \\ Ministry of Education, School of Information Science and Engineering, Department of Communication \\ Engineering, Xiamen University, Xiamen 361005, China; naveedrehmanjunejo@stu.xmu.edu.cn (N.U.R.J.); \\ zeyadqasem@stu.xmu.edu.cn (Z.A.H.Q.) \\ 2 Electrical Engineering Department, Faculty of Engineering, Aswan University, Aswan 81542, Egypt; \\ h.esmaiel@aswu.edu.eg \\ 3 Department of Information and Communication Engineering, School of Electrical and Electronic \\ Engineering, Tianjin University of Technology, Tianjin 300222, China; great_seal@163.com \\ * Correspondence: hxsun@xmu.edu.cn
}

Received: 22 April 2019; Accepted: 22 May 2019; Published: 24 May 2019

\begin{abstract}
Spatial Modulation Technologies (SMTs) are schemes that reduce inter-carrier interference (ICI), inter-channel interference, inter-antenna synchronization (IAS), and system complexity for multiple-input multiple-output (MIMO) communication systems. Moreover, high spectral and energy efficiency have rendered SMTs attractive to underwater acoustic (UWA) MIMO communication systems. Consequently, this paper focuses on SMTs such as spatial modulation (SM), generalized spatial modulation (GSM), and fully generalized spatial modulation (FGSM) in which one constant number and one multiple number of antennas are active to transmit data symbols in any time interval for underwater acoustic communication (UWAC). In SMTs, the receiver requires perfect channel state information (P-CSI) for accurate data detection. However, it is impractical that the perfect channel knowledge is available at the receiver. Therefore, channel estimation is of critical importance to obtain the CSI. This paper proposes the pilot-based recursive least-square (RLS) adaptive channel estimation method over the underwater time-varying MIMO channel. Furthermore, maximum likelihood (ML) decoder is used to detect the transmitted data and antennas indices from the received signal and the estimated UWA-MIMO channel. The numerical computation of mean square error (MSE) and bit error rate (BER) performance are computed for different SMTs like SM, GSM and FSGM using Monte Carlo iterations. Simulation results demonstrate that the RLS channel estimation method achieves the nearly same BER performance as P-CSI.
\end{abstract}

Keywords: Channel Estimation; MSE; BER; MIMO; ML; Spatial Modulation Technologies

\section{Introduction}

The enactment of UWA channels for reliable high data rate underwater wireless communication is very challenging due to the characteristics of the oceanic environment. The main communication technologies, including optical, magnetic induction, electromagnetic, and acoustic communications, are employed in UWCs. In optical communication, the salient features, including scattering, severe absorption, and LoS communication, make its applications limited to clean water only [1,2]. In magnetic and electromagnetic waves, harsh attenuation occurs via ocean conductivity, and ultra-low frequency bands overcome this attenuation. Acoustic communication technology is a widely trusted physical layer technology used in the UWC to achieve long communication distances, i.e., a few meters to 
tens of kilometers for low and high transmission frequencies. The acoustic wave is operated with limited bandwidth, ambient noise effects, propagation delay, and high Doppler shift [3,4]. Despite the problems mentioned earlier, acoustic communication is the most trusted UWC technology.

A MIMO system is deployed in underwater acoustic communication (UWAC) to overcome the UWC data rate limitations. In conventional UWA-MIMO systems, all transmission antennas are used in one time interval; hence, its performance depends on various parameters such as the distance between transmitter/receiver antennas [5,6], transmitter IAS, and receiver ICI $[7,8]$. The performance of the UWA-MIMO system can be degraded significantly due to incorrect IAS [7,8]. Despite this, UWA-MIMO technologies have brought a significant increase in capacity and diversity gain for acoustic underwater wireless networks [9] but there are many limitations such as implementation complexity due to multiple transmitter antennas, and performance deterioration due to the multipath propagation environment. In most of the MIMO systems, the implementation of a few transmitter antennas is led by power-loss problems [10]. Therefore, the SM scheme has been proposed in which only one transmitting antenna is activated in each time interval, and the possible data rate is compensated for by using spatial domain [11].

SM [12-14] is a unique method that accomplishes high spectral efficiency through spatial domain in MIMO transmission systems and information bits transmitted by only one antenna, providing higher data rate instead of traditional SIMO systems. The elementary principle of the SM is that it uses additional bits to convey the information through antenna indices and signal constellations such as M-QAM and M-PSK, where M is the constellation size. At the receiver, the SM decoder jointly searches all the transmitting antennas and M-ary constellation points to determine both the transmitted antenna index and the symbol. Therefore, SM has less receiver complexity, it effectively removes ICI, and it does not require IAS of the MIMO link due to availability of one RF chain at the transmitter. Different SMTs are introduced [10,15-23] by allowing it to enable one or more RF chains using different techniques so that high throughput and enhanced SNR can be achieved. GSM [21,22] is the most prevalent technique, in which the multiple antennas are active in each communication channel so that high data rate can be achieved. The activated antennas transmit the same symbol in each time interval to achieve transmitting diversity. Consequently, the available data rate of GSM is improved by activating a more significant number of transmitting antennas.

FGSM has been proposed in $[15,16]$ where the antenna subsets are varying between the activation of a single antenna and the activation of multiple/all antennas. These variations in the number of activated transmission antennas enhance the realization of the communication channel, resulting in increased available data rate. An additional parameter is also used to map the information bits, i.e., data signal constellations [17]. Therefore, the signal constellation type is also used to carry the transmitted data in addition to indices of the transmit antennas as in SM, GSM, and FGSM. The number of combinations between modulations and transmit antenna are increased by using the primary and secondary constellations. In this way, the receiver complexity is reduced, and performance gain of spectral efficiency is improved with high power saving.

In the SMTs, the receiver detects both active antenna/antennas index/indices and the transmitted symbol because it transmits the antenna index and transmitted information through the modulated signal. In the literature, the symbol detection and the antenna index are estimated by sub-optimal and optimal detection methods [12,24]. The error performance can be improved by using SM instead of V-BLAST in some cases, assuming that the receiver has complete CSI [24,25]. Practically, the receiver does not have the CSI, therefore, channel estimation has been performed to estimate the channel. In [26,27] SM-MIMO systems are tested to check the effect of rough channel estimation. In [26], MSE performance in MIMO systems is examined through LS estimation technique over quasi-static Rayleigh flat fading channels. Data detection with joint channel estimation is proposed with the assumption that the channel correlation matrix is available at the receiver [27]. To attain coherent detection performance, PSAM is applied in wireless communication [28]. The channel estimation for SM system through the RLS method based on pilots is investigated by assuming that for the duration 
of at least one length of frame the wireless channel is quasi-static [29]. In the case of the UWA channel, the channel estimation problem is more severe, where the union of a significant Doppler effects and long delay spread restrain UWA channels as doubly (time and frequency) spread channels [30,31].

In this work SM, GSM, and FGSM techniques are used to transmit the pilots and data symbols at time instant where one or multiple antennas are active. Therefore, CSI of the active transmit antennas can be obtained at the receiver by employing pilot-based channel estimation using the RLS channel estimation method. The performance of RLS improves if more pilots are employed for underwater time-varying channels, but unfortunately that affected the spectral efficiency. The challenging design for the MIMO system is to make pilot-transmitted sequences transmit from each antenna orthogonally to avoid inter-antenna interference. Spatially modulated MIMO systems handle this problem easily because transmitting antennas are mutual disjoints all the time, which makes each transmitted pilot sequence surely orthogonal to each other.

On the other hand, to the best of the authors' knowledge, no studies have been published to investigate the performance of SMTs with ML decoder using imperfect CSI in the UWA communication system. Therefore, in this paper, the RLS adaptive channel estimation is used in the ML optimal detector to detect the transmit data and antenna indices. Computer simulations are also performed to determine how the proposed adaptive channel estimation algorithm approaches the BER performance of P-CSI. For simplicity and ease for the reader, acronyms are mentioned in Table 1.

Table 1. Acronyms.

\begin{tabular}{ll}
\hline AWGN & Additive White Gaussian Noise \\
BER & Bit Error Rate \\
bpcu & Bit Per Channel Use \\
CSI & Channel State Information \\
EFGSM & Enhanced Fully Generalized Spatial Modulation \\
FGSM & Fully Generalized Spatial Modulation \\
GSM & Generalized Spatial Modulation \\
GSSK & Generalized Spatial Shift Keying \\
IAS & Inter-antenna Synchronization \\
ICI & Inter-carrier Interference \\
LMS & Least Mean Square \\
LoS & Line of Sight \\
LS & Least-Squares \\
MIMO & Multiple-Input Multiple-Output \\
ML & Maximum Likelihood \\
M-PSK & M-ary Phase Shift Keying \\
M-QAM & M-ary Quadrature Amplitude Modulation \\
MSE & Mean Square Error \\
P-CSI & Perfect Channel State Information \\
pdf & Probability Density Function \\
PSAM & Pilot Symbol Assisted Modulation \\
RF & Radio Frequency \\
RLS & Recursive Least-Squares \\
SIMO & Single-Input Multiple-Output \\
SM & Spatial Modulation \\
SMT & Spatial Modulation Technology \\
SNR & Signal to Noise Ratio \\
SSK & Spatial Shift Keying \\
UWA & Underwater Acoustic \\
UWAC & Underwater Acoustic Communication \\
UWC & Underwater Communication \\
V-BLAST & Vertical-Bell Laboratories Layered Space-Time \\
\hline & \\
\hline
\end{tabular}

The rest of the paper is managed as follows: The related work of SMTs is briefly explained in Section 2. Section 3 contains the ML optimal detector. The underwater channel model is presented in 
Section 4. In Section 5, RLS adaptive channel estimation algorithm described in brief. The simulation results and discussion are presented in Section 6. Finally, the paper is concluded in Section 7.

\section{Spatial Modulation Technologies}

In this section, the details of SMTs that can be used in the UWA are presented. First, the conventional SM is discussed in detail for underwater. Then, the underwater GSM and FGSM are explained in detail. Figure 1 shows the SMTs system model.

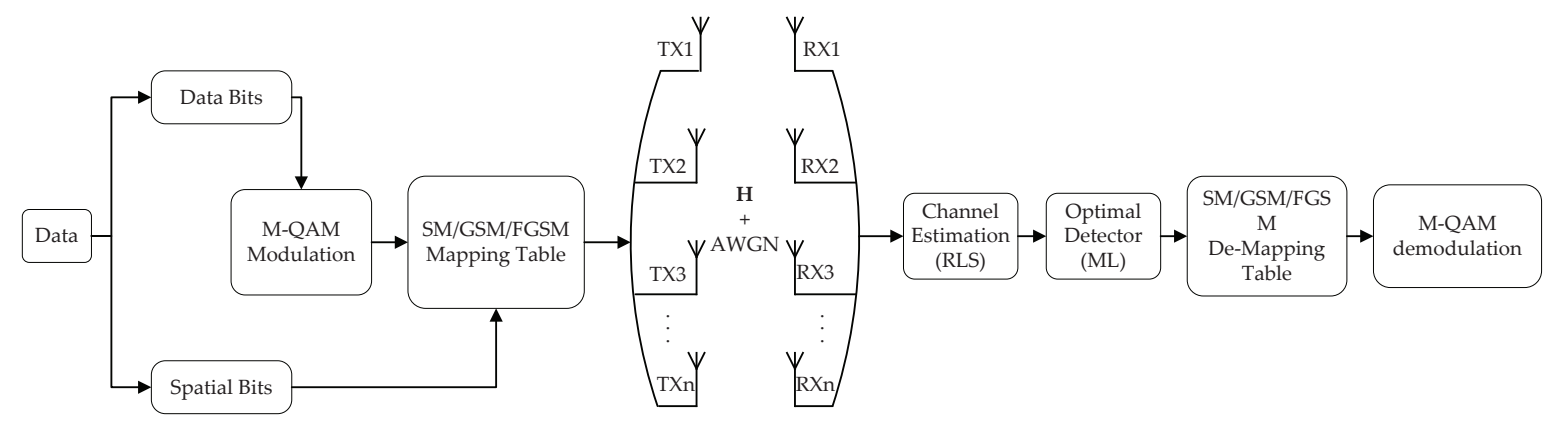

Figure 1. SMTs System Model.

\subsection{Spatial Modulation}

According to the current survey, the conception of SMTs is thought of as a hot topic among the top innovative researchers in the past decade. The easiest and most trivial version of SMT is the SSK [32-34], where information is embedded employing a transmit antenna index instead of using the constant amplitude/phase constellation symbols. SMTs do not require amplitude/phase elements to produce the data constellation symbols (for instance, RF chains are not required), which eventually relaxes the hardware need in the transmitter side, and in the computational complexity of the receiver-side detection. The achievable data rate of the SSK can be written as [32-34]:

$$
m_{S S K}=\log _{2}\left(N_{t}\right),
$$

where $N_{t}$ is the number of transmitting antennas.

The SM is a transmission technology that has been introduced for MIMO communication systems. The idea behind SM is to increase the total spectral efficiency so that the data is conveyed by both spatial antenna positions and amplitude/phase-modulation schemes. The spectral efficiency of SM increases in proportion to the base-two logarithm of the number of transmitting antennas. In SM, there is no need for the synchronization between the transmitting antennas because, at transmission instant, just one transmitting antenna from all transmitting antennas is active, while the others are off (only single RF chain is needed at transmitter side). Moreover, ICI is averted at the receiver, which results in low receiver complexity. At the receiver, the SM is used to estimate both transmitting antenna number and the sent symbol; after that, the spatial demodulator is used to restore the original data bits [18]. In the SM, input binary bits are split into two sets-the index sets and the constellation sets. The index of an active transmit antenna chosen by the set of $\log _{2}\left(N_{t}\right)$ bits and the second set of bits is $\log _{2}(M)$ chooses the transmit symbol from M-ary signal constellation. In the SM system, the number of transmitted data bits $m$ can be varied by signal and spatial modulation. For example, in the SM system, four bits can be transmitted two ways to achieve the same spectral efficiency; one is using two transmitting antennas and 8-QAM modulation, and in the second, four transmitting antennas and 4-QAM can be used. Generally, the spectral efficiency of SM is given as follows [12].

$$
m_{S M}=\log _{2}(M)+\log _{2}\left(N_{t}\right)
$$


SM maps a random stream of $m$ bits at different symbol intervals into $N_{t}$-dimensional signal as:

$$
\mathbf{x}=\left[x_{1}, x_{2}, \ldots x_{N_{t}}\right]^{T} .
$$

Only one of $\mathbf{x}_{g}$ that is active in $\mathbf{x}$ is nonzero, where $g$ represents the index of active antenna. Then, at the $k^{\text {th }}$ symbol interval, at the SM-MIMO transmitter the resultant output transmit vector can be represented as:

$$
\mathbf{x}_{g} \triangleq\left[0 \ldots x_{k} \ldots 0\right]^{T},
$$

where $x_{k}$ denotes the $k^{\text {th }}$ symbol from the M-ary constellation diagram. Over this symbol duration, the other antennas will not be active. The symbol $x_{k}$ is the transmitted data symbol over an $N_{r} \times N_{t}$ MIMO channel from antenna $g$.

The SM mapping procedure is revealed in Table 2, for $4 \mathrm{bpcu}$, where $N_{t}=4$ and 4-QAM based on the gray-coded constellation points. Consider that the bit stream of [10 11] at any time instant is transmitted through the SM system. The bit stream is split into two parts where the first group of two bits [10] is opted to select the data symbol from the constellation. The second group of bits [11] is used as the spatial bits that chooses the antenna for symbol transmission from the antenna combination given in Table 2. As a result, the transmit vector for SM-MIMO system becomes:

$$
\mathbf{x}_{g} \triangleq\left[\begin{array}{llll}
0 & 0 & x_{k} & 0
\end{array}\right]
$$

Table 2. Example of SM mapping for $4 \mathrm{bpcu}, N_{t}=4$ and 4-QAM modulation.

\begin{tabular}{cccc}
\hline Block Bits & Spatial Bits & Data Bits & Antenna Index \\
\hline $00 \mathrm{~b} 1 \mathrm{~b} 2$ & 00 & $\mathrm{~b} 1 \mathrm{~b} 2$ & 1 \\
$01 \mathrm{~b} 1 \mathrm{~b} 2$ & 01 & $\mathrm{~b} 1 \mathrm{~b} 2$ & 2 \\
$10 \mathrm{~b} 1 \mathrm{~b} 2$ & 10 & $\mathrm{~b} 1 \mathrm{~b} 2$ & 3 \\
$11 \mathrm{~b} 1 \mathrm{~b} 2$ & 11 & $\mathrm{~b} 1 \mathrm{~b} 2$ & 4 \\
\hline
\end{tabular}

\subsection{Generalized Spatial Modulation}

GSSK $[35,36]$ is related to SSK, but GSSK does not require the generation of the habitual data constellations symbols. However, the transmit antennas are used to embed information in GSSK. In SSK a single transmitting antenna is operated to transfer information. Therefore, RF chains are no more required in the transmission side for GSSK. Moreover, to route the transmitted data, the single RF splitter is entailed to the corresponding transmit antennas using RF switches. Mathematically, the GSSK data rate is stated as follows [35,36]:

$$
m_{G S S K}=\left\lfloor\log _{2}\left(\begin{array}{c}
N_{t} \\
N_{a}
\end{array}\right)\right\rfloor,
$$

where $N_{a}$ is the number of active antennas for data transmission, $\left(\begin{array}{c}. \\ \cdot\end{array}\right)$ represents the binomial coefficient and $\lfloor$.$\rfloor represents the floor operator.$

The GSM $[21,22,37,38]$ is the combination of GSSK and SM that attains spectral efficiency higher than SM by activating a greater number of antennas, and it only requires a single RF chain for data transmission. The mechanism of GSM is similar to SM, in which the data is mapped by antenna indices and the symbol constellation. In addition, the same symbol data is transmitted from the active antennas. Due to the activation of a higher number of transmitting antennas; GSM achieves a higher data rate than the $\mathrm{SM}$, and its achievable data rate is stated as follows [21,22,37,38]: 


$$
m_{G S M}=\log _{2}(M)+\left\lfloor\log _{2}\left(\begin{array}{c}
N_{t} \\
N_{a}
\end{array}\right)\right\rfloor .
$$

The GSM mapping procedure is revealed in Table 3, for $4 \mathrm{bpcu}$, and is fulfilled by using $N_{t}=4$ and 4-QAM based on the gray-coded constellation points. Consider the bit stream of [10 00] at any time instant is transmitted through the GSM system. The bit stream is split into two parts where the first group of two bits [10] is opted to select the data symbol from the constellation. The second group of bits [00] is used as the spatial bits that chooses the antenna for symbol transmission from the antenna combination given in Table 3. As such, the resultant transmission vector of the GSM-MIMO system is presented as:

$$
\mathbf{x}_{g} \triangleq\left[\begin{array}{llll}
x_{k} & x_{k} & 0 & 0
\end{array}\right]
$$

Table 3. Example of GSM mapping for $4 \mathrm{bpcu}, N_{t}=4, N_{a}=2$ and 4-QAM modulation.

\begin{tabular}{cccc}
\hline Block Bits & Spatial Bits & Data Bits & Antenna Index \\
\hline $00 \mathrm{~b} 1 \mathrm{~b} 2$ & 00 & $\mathrm{~b} 1 \mathrm{~b} 2$ & 1,2 \\
$01 \mathrm{~b} 1 \mathrm{~b} 2$ & 01 & $\mathrm{~b} 1 \mathrm{~b} 2$ & 1,3 \\
$10 \mathrm{~b} 1 \mathrm{~b} 2$ & 10 & $\mathrm{~b} 1 \mathrm{~b} 2$ & 1,4 \\
$11 \mathrm{~b} 1 \mathrm{~b} 2$ & 11 & $\mathrm{~b} 1 \mathrm{~b} 2$ & 2,3 \\
\hline
\end{tabular}

In GSM, the same symbol transmission from active antennas in one time instant has no hard bound dictating that the number of transmitting antennas should be a power of two, but an absolute number of transmitting antennas can be employed. The GSM attains a spectral efficiency higher than SM with a remarkably lower number of transmitting antennas, and with a slight increment in transmitter complexity. Furthermore, this complexity gain is due to the number of active antennas. To reach the spectral efficiency similar to the GSM, SM needs a greater number of transmitting antennas than GSM at the same modulation order.

\subsection{Fully Generalized Spatial Modulation}

Based on the GSM scheme, the FGSM has been proposed $[15,16]$. In the FGSM, a block of transmitted bit instants is divided into two different groups, namely data bits and spatial bits. The data bits embed $\log _{2}(M)$, which is to modulate a signal constellation symbol from a signal constellation diagram of an arbitrary M-QAM or any other signal constellation diagram. The spatial bits embed $N_{t}-1$ bits and are employed to choose the antenna subset to transmit the data constellation symbols. In FGSM, the antenna subsets are changed in a way that the active antennas can be one or multiple/all transmit antennas. This is entirely against conventional technologies in the SM and GSM, because in SM and GSM a single or a constant number of antennas are being used, respectively. Changing the number of active transmit antennas in the antenna subsets improves the understanding of the communication channel, simplifies the procedure of differentiating between the channel's multiple paths, and mitigates the weakness in the performance of BER. Hence, the FGSM available data rate can be expressed as follows $[15,16]$ :

$$
m_{F G S M}=\log _{2}(M)+\left\lfloor\log _{2}\left(2^{N_{t}-1}\right)\right\rfloor=\log _{2}(M)+\left(N_{t}-1\right) .
$$

The principal transmission of the FGSM is exemplified in Table 4. FGSM achieves the spectral efficiency of 5 bpcu using 4 transmit antennas and 4-QAM based on the gray-coded constellation points. However, 8 transmit antennas are essential in SM to achieve this spectral efficiency, and in GSM 5 transmit antennas with $N_{a}=3$ are needed. Consider if the bit stream of [10 111] at any time instant is transmitted through the FGSM system. The bit stream is split into two parts where the first 
group of two bits [10] is opted to select the data symbol from the constellation. The second group of bits [111] is used as the spatial bits that chooses antenna for symbol transmission from the antenna combination given in Table 4. As a result, the transmit vector for FGSM-MIMO system becomes:

$$
\mathbf{x}_{g} \triangleq\left[\begin{array}{llll}
0 & x_{k} & x_{k} & 0
\end{array}\right]
$$

Table 4. Example of FGSM mapping for $5 \mathrm{bpcu}, \mathrm{N}_{t}=4$ and 4-QAM modulation.

\begin{tabular}{cccc}
\hline Block Bits & Spatial Bits & Data Bits & Antenna Index \\
\hline $000 \mathrm{~b} 1 \mathrm{~b} 2$ & 000 & $\mathrm{~b} 1 \mathrm{~b} 2$ & 1 \\
$001 \mathrm{~b} 1 \mathrm{~b} 2$ & 001 & $\mathrm{~b} 1 \mathrm{~b} 2$ & 2 \\
$010 \mathrm{~b} 1 \mathrm{~b} 2$ & 010 & $\mathrm{~b} 1 \mathrm{~b} 2$ & 3 \\
$011 \mathrm{~b} 1 \mathrm{~b} 2$ & 011 & $\mathrm{~b} 1 \mathrm{~b} 2$ & 4 \\
$100 \mathrm{~b} 1 \mathrm{~b} 2$ & 100 & $\mathrm{~b} 1 \mathrm{~b} 2$ & 1,2 \\
$101 \mathrm{~b} 1 \mathrm{~b} 2$ & 101 & $\mathrm{~b} 1 \mathrm{~b} 2$ & 1,3 \\
$110 \mathrm{~b} 1 \mathrm{~b} 2$ & 110 & $\mathrm{~b} 1 \mathrm{~b} 2$ & 1,4 \\
$111 \mathrm{~b} 1 \mathrm{~b} 2$ & 111 & $\mathrm{~b} 1 \mathrm{~b} 2$ & 2,3 \\
\hline
\end{tabular}

\section{Optimal Detector}

Recently, the FGSM [15] and EFGSM [39] have been proposed to the UWA channel to improve the energy loss of the underwater sensor network. The transmission vector of the SMTs-MIMO is transmitted over underwater time-varying MIMO channel with an AWGN. As a result, the received signal after MIMO channel at the receiver can be represented as:

$$
\mathbf{y}=\mathbf{H x}+\mathbf{v},
$$

where $\mathbf{y}$ is the received data $\mathbf{y} \in \mathbb{C}^{N_{r} \times 1}, \mathbf{H}$ denotes the UWA channel $\mathbf{H} \in \mathbb{C}^{N_{r} \times N_{t}}, \mathbf{x}$ is the sending data mapped by SMTs $\mathbf{x} \in \mathbb{C}^{N_{t} \times 1}$ and an AWGN $\mathbf{v} \in \mathbb{C}^{N_{r} \times 1}$ with identically independent (i.i.d) Gaussian distributed represented as $\mathbf{v} \sim C N\left(0, \sigma^{2}\right)$, and $N_{r}$ is the number of receiver antennas, where $\mathbf{v}$ is the complex value of the AWGN, and can be written as:

$$
\mathbf{v}=\left[v_{1}, v_{2}, \ldots, v_{N_{r}}\right]^{T} .
$$

Hence, Equation (11) can be written as:

$$
\left[\begin{array}{c}
y_{1} \\
y_{2} \\
\vdots \\
y_{N_{r}}
\end{array}\right]=\left[\begin{array}{cccc}
\mathbf{h}_{11} & \mathbf{h}_{12} & \ldots & \mathbf{h}_{1 N_{t}} \\
\mathbf{h}_{21} & \mathbf{h}_{22} & \ldots & \mathbf{h}_{2 N_{t}} \\
\vdots & \vdots & \ddots & \vdots \\
\mathbf{h}_{N_{r} 1} & \mathbf{h}_{N_{r} 2} & \ldots & \mathbf{h}_{N_{r} N_{t}}
\end{array}\right] \times\left[\begin{array}{c}
x_{1} \\
x_{2} \\
\vdots \\
x_{N_{t}}
\end{array}\right]+\left[\begin{array}{c}
v_{1} \\
v_{2} \\
\vdots \\
v_{N_{r}} .
\end{array}\right] .
$$

The detection of antenna indices is a very challenging task in SMTs, in SM, GSM and FGSM antennas active are one constant number of antennas, and one or more/all, respectively. Among the set of transmit antennas, to detect the transmitted data, the antenna indices must be decided at the receiver side. The conventional ML optimal detector is used in the SMTs to detect the data constellation and antenna indices, and its representation equation can be written as [24]:

$$
\left[\widehat{g}_{M L}, \widehat{k}_{M L}\right]=\underset{g, k}{\arg \max } p_{Y}\left(\mathbf{y} \mid \mathbf{x}_{g}, \mathbf{H}\right) .
$$

The $\mathbf{x}_{g}$ is the $k^{\text {th }}$ data symbol from M-ary constellation transmitted over the channel $\mathbf{h}_{g}$ which represents the $g^{\text {th }}$ row of the matrix channel $\mathbf{H}$. As we can see in (5), (8), and (10) $\mathbf{x}_{g}$ varies for different $g$ and $k$. The pdf conditioned to $\mathbf{x}_{g}$ and $\mathbf{h}_{g}$ of $\mathbf{y}$ in Equation (14) can be stated as: 


$$
p_{Y}\left(\mathbf{y} \mid \mathbf{x}_{g}, \mathbf{H}\right)=\pi^{-N_{r}} \exp \left(-\left\|\mathbf{y}-\mathbf{h}_{g} x_{k}\right\|_{F}^{2}\right),
$$

where

$$
\mathbf{h}_{g}=\left[h_{g 1}, h_{g 2}, \ldots, h_{g N_{t}}\right] .
$$

After using (14) the optimal detector can be represented as:

$$
\left[\hat{g}_{M L}, \hat{k}_{M L}\right]=\underset{g, k}{\arg \min }\left\|\mathbf{j}_{g k}\right\|_{F}^{2}-2 \Re\left\{\mathbf{y}^{H} \mathbf{j}_{g k}\right\},
$$

where $\mathbf{j}_{g k}=\mathbf{h}_{g} x_{k}, 1 \leq g \leq N_{t}, 1 \leq k \leq M$ and $\Re\{$.$\} is the real operator. If both \widehat{g}_{M L}$ and $\widehat{k}_{M L}$ is detected correctly by the receiver, then they can be demapped easily and combined to demodulate the transmitting bits. For accurate detection, at the receiver side the completed CSI should be available.

\section{Underwater Acoustic Channel Model}

The SMTs have been applied for the UWA channel in $[15,39]$ considering the full channel information at the receiver side. Unfortunately, the UWA channel is hard to adequate estimate; in this section we will address the UWA features. The impulse response of the time-varying multipath UWA channel can be determined as [30,40,41]:

$$
\mathbf{H}(t, \tau)=\sum_{c} A_{c}(t) \delta\left(\tau-\tau_{c}(t)\right),
$$

where $\tau_{c}(t)$ stands for the delay of the time-varying path and $\left.A_{c}(t)\right)$ is the path amplitude. The given channel comprises several essential pathways; every pathway is scrutinized by time-varying pathway amplitude and delay information. We further assume the below-mentioned conditions for simplifying the receiver processing algorithms and developing while maintaining generality. The delays in time-varying channels are sparked by the movement of the transmitter as well as receiver along with scattering from the sea surface or refraction caused by sound-speed variability. The path amplitude varies with the delay as the attenuation is attributed to the traveled distance and the physics associated with the scattering phenomenon. Assuming the path amplitude $\left.A_{c}(t)\right)$ remains consistent during the transmission of the same block:

$$
A_{c}(t)=A_{c},
$$

and during the data symbol transmission all paths delays $\tau_{c}(t)$ have a similar Doppler-scaling factor as:

$$
\tau_{c}(t)=\tau_{c}-\beta t,
$$

where $\beta$ is the Doppler-scaling factor. Generally, each path has its own Doppler-scaling factor, but herein, we have considered the similar Doppler-scaling factor for each path. Moreover, we recommend the approximation of the underwater acoustic channel by $\rho$ dominant discrete paths, so the UWA channel can be presented as:

$$
\mathbf{H}(t, \tau)=\sum_{c}^{\rho} A_{c}(t) \delta\left(\tau-\left(\tau_{c}-\beta t\right)\right) .
$$

\section{Recursive Least-Squares Adaptive Channel Estimation}

RLS channel estimation serves as a distinct adaptive filter method employed for finding the coefficients recursively to reduce the cost function of a weighted linear least-squares corresponding to the input signal. The mentioned algorithm is highly contrasting to the other algorithms, just as LMS minimizes the MSE. This paper uses the RLS method in SMTs for channel estimation using pilot symbols, which is represented by $\mathbf{x}_{P, i}$. The pilot symbols initially undergo sequential transmission via transmit antennas along with the channel coefficients for the optimum transmission as well as antenna receiver, and then pilots are estimated by the proposed algorithm on an individual level for the whole 
duration of every pilot symbol. In this work, the received signal pilot symbols are represented by $\mathbf{y}_{r_{x}, P, i}$, where $r_{x}=1,2, \ldots, N_{r}$ and $P$ is pilot. The received signals go through sequential processing at the receiver end, and the up-gradation of channel estimations is carried out upon the arrival of new samples. This section briefly illustrates the recursive computation of least-squares channel estimations. The transmitted pilot symbols can be written as:

$$
\mathbf{x}_{P, i}=\left[x_{P, 1}, x_{P, 2}, \ldots, x_{P, N_{P}}\right],
$$

where $N_{P}$ is the total number of pilots for active antennas. The received pilots from sub-channels of active antennas can be represented as:

$$
\mathbf{y}_{r_{x}, P, i}=\left[y_{r_{x}, P, 1}, y_{r_{x}, P, 2}, \ldots, y_{r_{x}, P, N_{P}}\right] .
$$

The set of linear filters output are defined by the following equation:

$$
\Im_{r_{x}, g, i}=\widehat{\mathbf{h}}_{r_{x}, g, i} \mathbf{x}_{P, i}, \quad i=1,2, \ldots, N_{P}
$$

where $\widehat{h}_{r_{x}, g, i}$ are the channel coefficients between the $g^{\text {th }}$ transmitted antennas and received antennas $r_{x}$ which are recursively estimated to minimize the sum of square errors denoted as:

$$
e_{i}=\sum_{n=1}^{i} \lambda\left(y_{r_{x}, P, n}-\widehat{\mathbf{h}}_{r_{x}, g, n} x_{P, n}\right)^{2},
$$

where $\lambda$ is the weighting factor, which reduces the interference of the old data and its selected value $0<\lambda \leq 1$. Figure 2 shows the basic adaptive channel estimation model which is used to estimate the UWA-MIMO channel of the SMTs. Here, $u_{i}$ is the input of the unknown sampled system as well as an adaptive filter. The response of the unknown sample system $d_{i}$ is measured in the presence of noise $v$. The adaptive filter response $y_{i}$ is subtracted from the unknown sample system output $d_{i}$. The error signal $e_{i}$ is the difference of adaptive filter and unknown sample system outputs, which is used for updating the adaptive filter coefficients. If the system is correctly devised, the transfer function of the adaptive filter will converge to the transfer function of the unknown sample system after a known number of Monte Carlo iterations. Here, the adaptive filter has been employed to estimate the coefficients of the UWA-MIMO channel using the RLS algorithm. The complexity of adaptive filter RLS is $O\left(N^{2}\right)$ per iteration, where $N$ is the filter size, but it is well known because of fast convergence [42,43]. The RLS steps are defined in Algorithm 1.

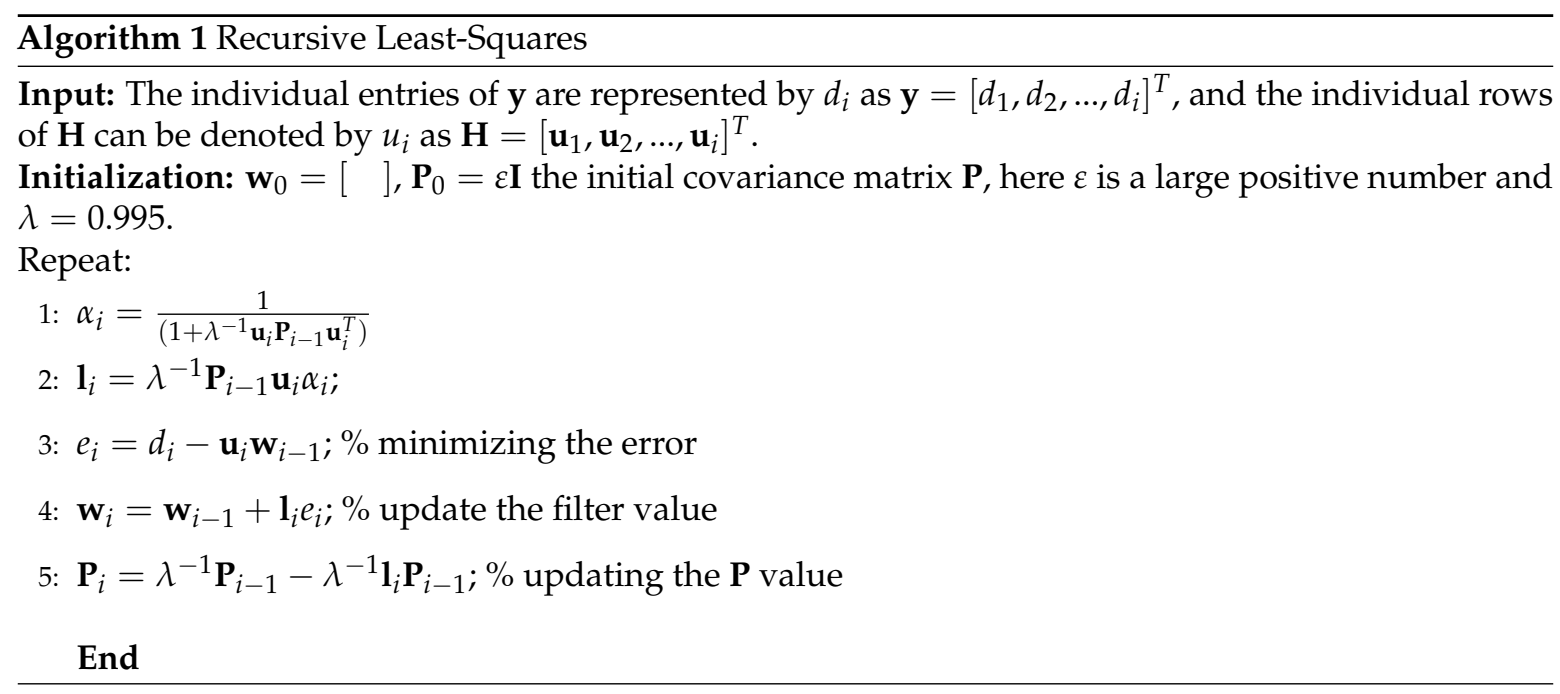

Here is the derivative of the Recursive Least-Squares,

$$
\boldsymbol{\phi}_{i}=\lambda \boldsymbol{\phi}_{i-1}+\mathbf{u}_{i} \mathbf{u}_{i}^{T}
$$


By applying the matrix inverse formula Equation (26) can be written as:

$$
\boldsymbol{\phi}_{i}^{-1}=\lambda^{-1} \boldsymbol{\phi}_{i}^{-1}-\frac{\lambda^{-2} \boldsymbol{\phi}_{i-1}^{-1} \mathbf{u}_{i} \mathbf{u}_{i}^{T} \boldsymbol{\phi}_{i-1}^{-1}}{1+\lambda^{-1} \mathbf{u}_{i}^{T} \boldsymbol{\phi}_{i-1}^{-1} \mathbf{u}_{i}}
$$

Replacing $\mathbf{P}_{i}=\boldsymbol{\phi}_{i}^{-1}$, the above equation can be expressed as:

$$
\mathbf{P}_{i}=\lambda^{-1} \mathbf{P}_{i-1}-\frac{\lambda^{-2} \mathbf{P}_{i-1} \mathbf{u}_{i} \mathbf{u}_{i}^{T} \mathbf{P}_{i-1}}{1+\lambda^{-1} \mathbf{u}_{i}^{T} \mathbf{P}_{i-1} \mathbf{u}_{i}}
$$

By simplifying the above equation, we get:

$$
\mathbf{P}_{i}=\lambda^{-1} \mathbf{P}_{i-1}-\lambda^{-1} \mathbf{l}_{i} \mathbf{P}_{i-1} .
$$

where $\mathbf{l}_{i}=\frac{\lambda^{-1} \mathbf{P}_{i-1} \mathbf{u}_{i}}{1+\lambda^{-1} \mathbf{u}_{i}^{T} \mathbf{P}_{i-1} \mathbf{u}_{i}}$, after further simplification we get:

$$
\mathbf{1}_{i}=\lambda^{-1} \mathbf{P}_{i-1} \mathbf{u}_{i} \alpha_{i}
$$

where $\alpha_{i}=\frac{1}{1+\lambda^{-1} \mathbf{u}_{i} \mathbf{P}_{i-1} \mathbf{u}_{i}^{T}}$, the main equation of the weighting factor $\mathbf{w}_{i}$ is derived as:

$$
\begin{aligned}
& \mathbf{w}_{i}=\mathbf{P}_{i} \lambda \boldsymbol{\phi}_{i-1} \mathbf{w}_{i-1}+\mathbf{u}_{i} d_{i}=\mathbf{P}_{i}\left(\left(\boldsymbol{\phi}_{i}-\mathbf{u}_{i} \mathbf{u}_{i}^{T}\right) \mathbf{w}_{i-1}+\mathbf{u}_{i} d_{i}\right)=\mathbf{w}_{i-1}-\mathbf{P}_{i} \mathbf{u}_{i} \mathbf{u}_{i}^{T} \mathbf{w}_{i-1}+\mathbf{P}_{i} \mathbf{u}_{i} d_{i} \\
& =\mathbf{w}_{i-1}+\mathbf{P}_{i} \mathbf{u}_{i} d_{i}-\mathbf{u}_{i}^{T} \mathbf{w}_{i-1}=\mathbf{w}_{i-1}+\mathbf{P}_{i} \mathbf{u}_{i} e_{i}=\mathbf{w}_{i-1}+\mathbf{l}_{i} e_{i}
\end{aligned}
$$

where

$$
e_{i}=d_{i}-\mathbf{u}_{i} \mathbf{w}_{i-1}
$$

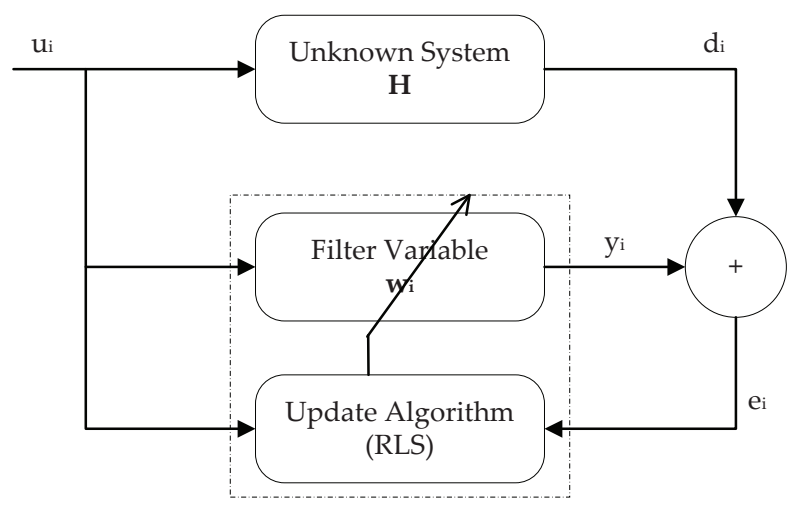

Figure 2. RLS Adaptive Channel Estimation Model.

\section{Simulation Results and Discussion}

In this section, the BER performance of the proposed RLS channel estimator is provided for SM, GSM, and FGSM over underwater time-varying acoustic MIMO channel. The simulation outcomes are achieved for $10^{6}$ symbols and transmitted over a regular underwater acoustic channel. For the transmission antennas, the total transmitting power is normalized. The uncorrelated data symbols are generated, and signal constellation has been chosen for data modulation. BER performance was computed for different SMTs such as SM, GSM, and FSGM using Monte Carlo iterations, where the channel is estimated by different pilot numbers compared with P-CSI. After channel estimation, the antenna indices and transmitted data symbols is estimated by using the ML optimal detector. For our computer simulations, two benchmarks are compared: (1) the BER performance in case of P-CSI; and (2) BER performance at a different number of pilots used for RLS channel estimation. The parameters chosen for our proposed RLS channel estimation methods are $\mathbf{P}_{0}=\varepsilon \mathbf{I}$, 
$\varepsilon=10^{6}, \lambda=0.995$, number of pilots are $2 \%, 5 \%, 10 \%$ and $15 \%$. The SNR is defined as $E_{s} / \sigma^{2}, E_{s}$ is energy per symbol and $\sigma^{2}$ is noise variance assuming that each receiving antenna has same power. A 16-QAM modulation and the channel $4 \times 4$ time-varying UWA-MIMO channel with normalized 0.15 Doppler-scaling factor is used for all SMT schemes. The spectral efficiency of the FGSM is $7 \mathrm{bpcu}$, and for SM/GSM it is 6 bpcu. In these simulation results, two scenarios are considered: the first is in the case of ignoring the effect of the Doppler-scaling factor of the UWA channel, and the second is holding the effect of the Doppler-scaling factor of the UWA channel. The MSE can be calculated as:

$$
M S E=\frac{\mathbb{E}\left\{\sum_{n}\left|\widehat{\mathbf{H}}_{n}-\mathbf{H}_{n}\right|^{2}\right\}}{\mathbb{E}\left\{\sum_{n}\left|\widehat{\mathbf{H}}_{n}\right|^{2}\right\}} .
$$

\subsection{Spatial Modulation}

Figure 3 shows the BER performance comparison of P-CSI with the RLS channel estimator using the different number of pilots for SM without Doppler-scaling factor over time-varying UWA-MIMO channel. The performance of RLS channel estimator using $2 \%$ of the transmitted information bits as a pilot is totally degraded as compared to higher-order pilot numbers. In Figure 3, the performance gap between the P-CSI and RLS channel estimator using $5 \%$ of pilots at BER $10^{-2}$ is $1.1 \mathrm{~dB}$. Therefore, a greater number of pilots are required to achieve the same BER performance as in the P-CSI. Furthermore, the performance gap between P-CSI and RLS channel estimator using $10 \%$ and $15 \%$ pilots is $0.18 \mathrm{~dB}$ and $0.09 \mathrm{~dB}$ at BER $10^{-2}$. Figure 3 revealed the BER performance of the RLS channel estimator approaches to P-CSI by increasing the number of pilots.

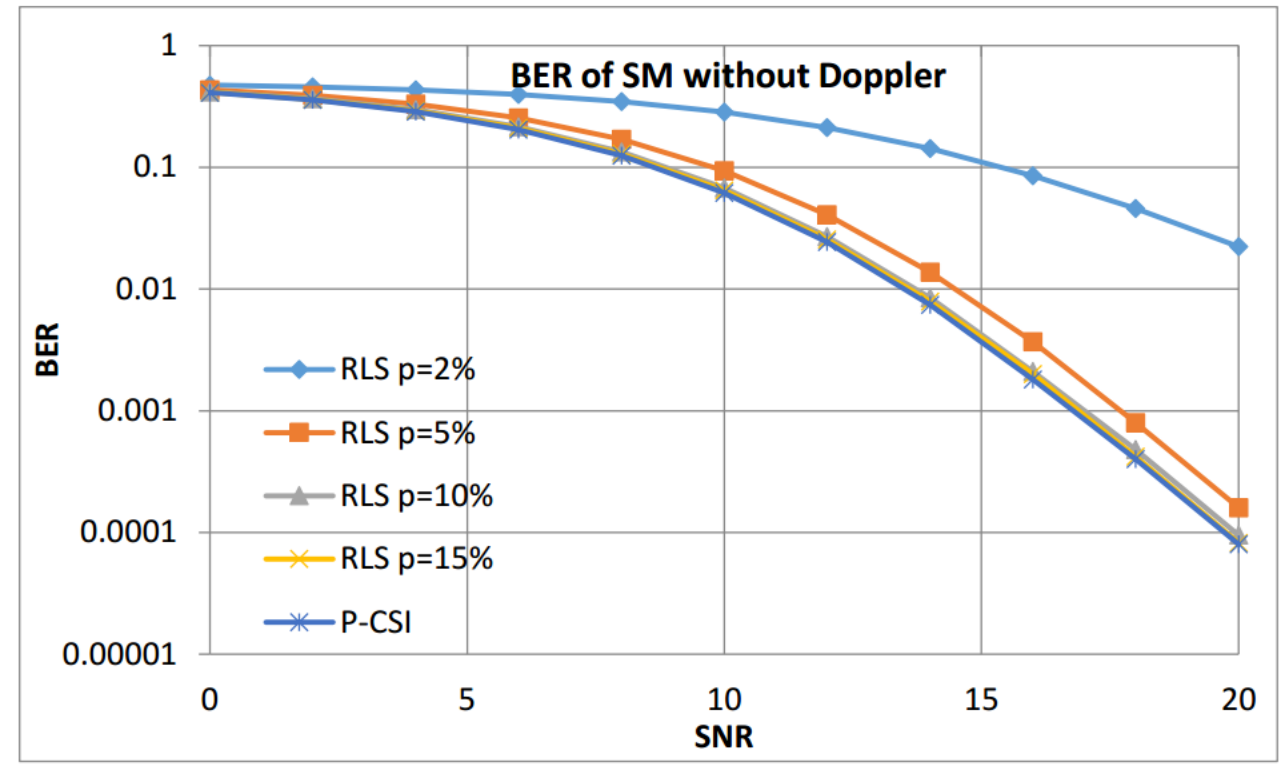

Figure 3. BER performance of the $4 \times 4$ underwater SM-MIMO without Doppler-scaling factor.

In Figure 4 shows the BER performance of SM by RLS estimator method using a different number of pilots compared with a P-CSI over time-varying UWA-MIMO channel with normalized 0.15 Doppler-scaling factor. In Figure 4 at BER $10^{-2}$ P-CSI demonstrates $10 \mathrm{~dB}, 1.22 \mathrm{~dB}, 0.2 \mathrm{~dB}$, and $0.11 \mathrm{~dB}$ better performance than RLS channel estimation using $2 \%, 5 \%, 10 \%$, and $15 \%$ pilots, respectively. From Figures 3 and 4, it is concluded that the SNR gain difference using RLS channel estimation with different number of pilots compared with P-CSI is almost the same for both with and without Doppler-scaling factor scenario. 


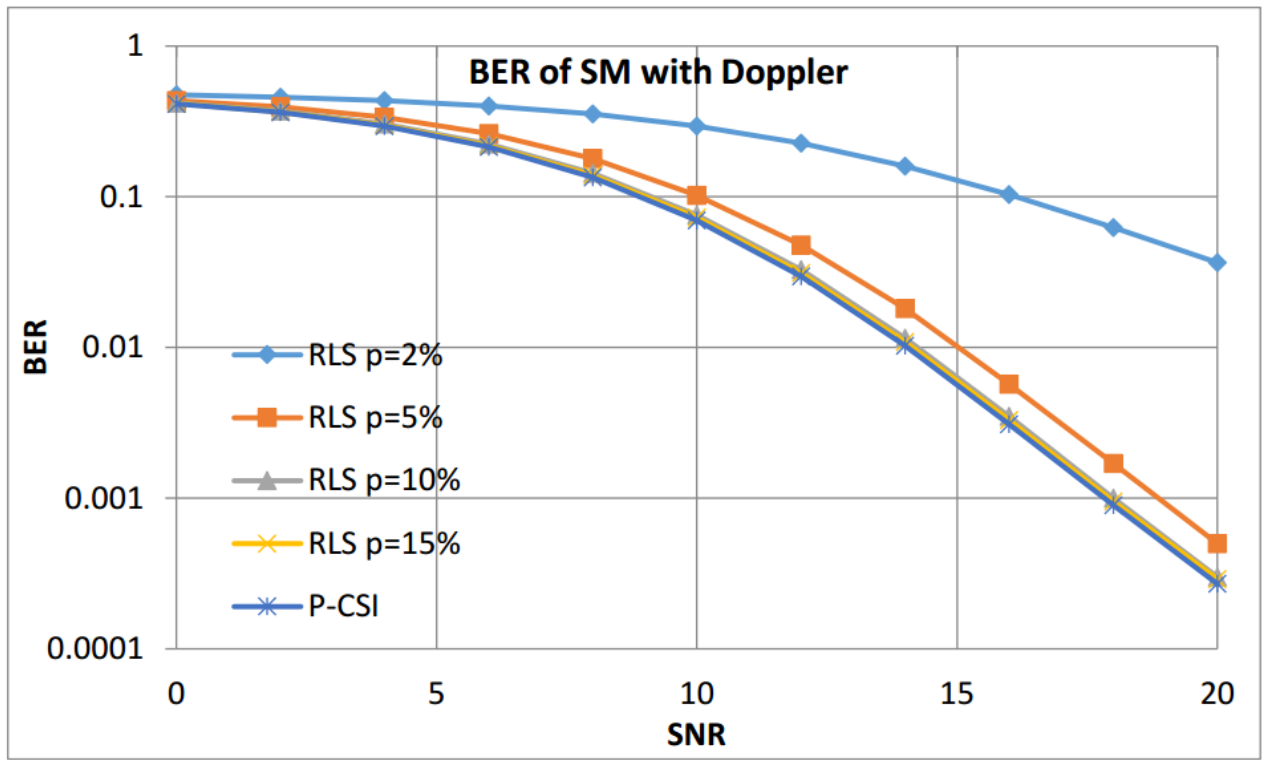

Figure 4. BER performance of the $4 \times 4$ underwater SM-MIMO with Doppler-scaling factor.

\subsection{Generalized Spatial Modulation}

Figure 5 illustrates the BER performance of GSM for P-CSI and estimated channel using the RLS estimation method for different numbers of pilots by ignoring the Doppler-scaling factor. The number of pilots is assumed to be $2 \%, 5 \%, 10 \%$, and $15 \%$ for time-varying UWA-MIMO channel estimation. The BER performance gain using $2 \%$ of pilots using RLS channel estimator is too low with a difference of $9 \mathrm{~dB}$ at BER $10^{-2}$. To attain nearly the same performance as P-CSI, a greater number of pilots are used for the RLS channel estimation method. In comparison to the P-CSI at the target of $10^{-2}$, BER the SNR gain difference is $1.75 \mathrm{~dB}, 0.28 \mathrm{~dB}$, and $0.15 \mathrm{~dB}$ for $5 \%, 10 \%$, and $15 \%$ pilots using RLS channel estimator, respectively. Figure 5 clarifies that the SNR gain between P-CSI and RSL channel estimation using $10 \%$ and $15 \%$ pilots is almost negligible.

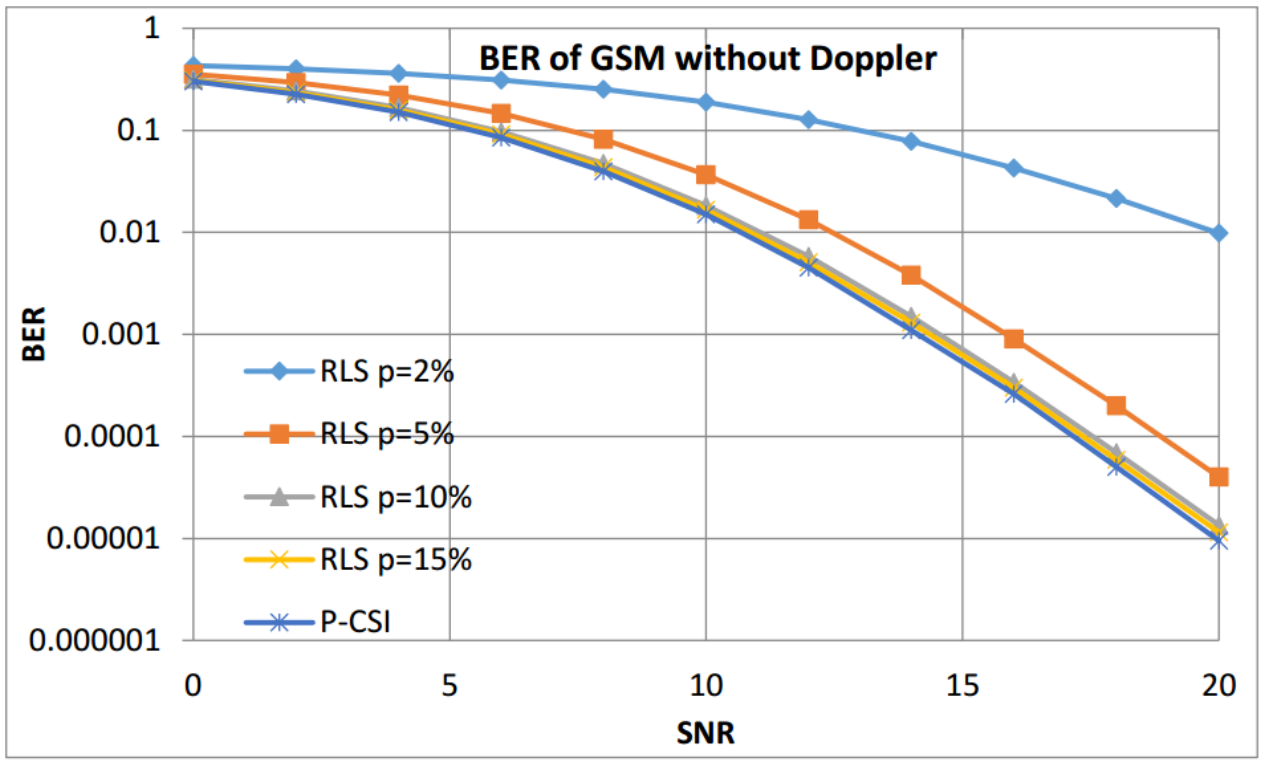

Figure 5. BER performance of the $4 \times 4$ underwater GSM-MIMO without Doppler-scaling factor.

The BER performance of RLS channel estimation method using pilots compared with a P-CSI for GSM over time-varying UWA-MIMO channel with Doppler-scaling factor is demonstrated in Figure 6. 
At BER $10^{-2}$ the SNR difference gain is $1.78 \mathrm{~dB}$ for $5 \%$ of pilots, $0.31 \mathrm{~dB}$ using pilots $10 \%$, and $0.21 \mathrm{~dB}$ for $15 \%$ as compared to P-CSI. The SNR gain difference becomes very small with P-CSI while pilots are increased from $5 \%$ to $10 \%$ and $15 \%$ in presence of Doppler effect. However, it can be seen from Figure 6 that there is huge performance gap between RLS channel estimator using $2 \%$ of pilots and P-CSI.

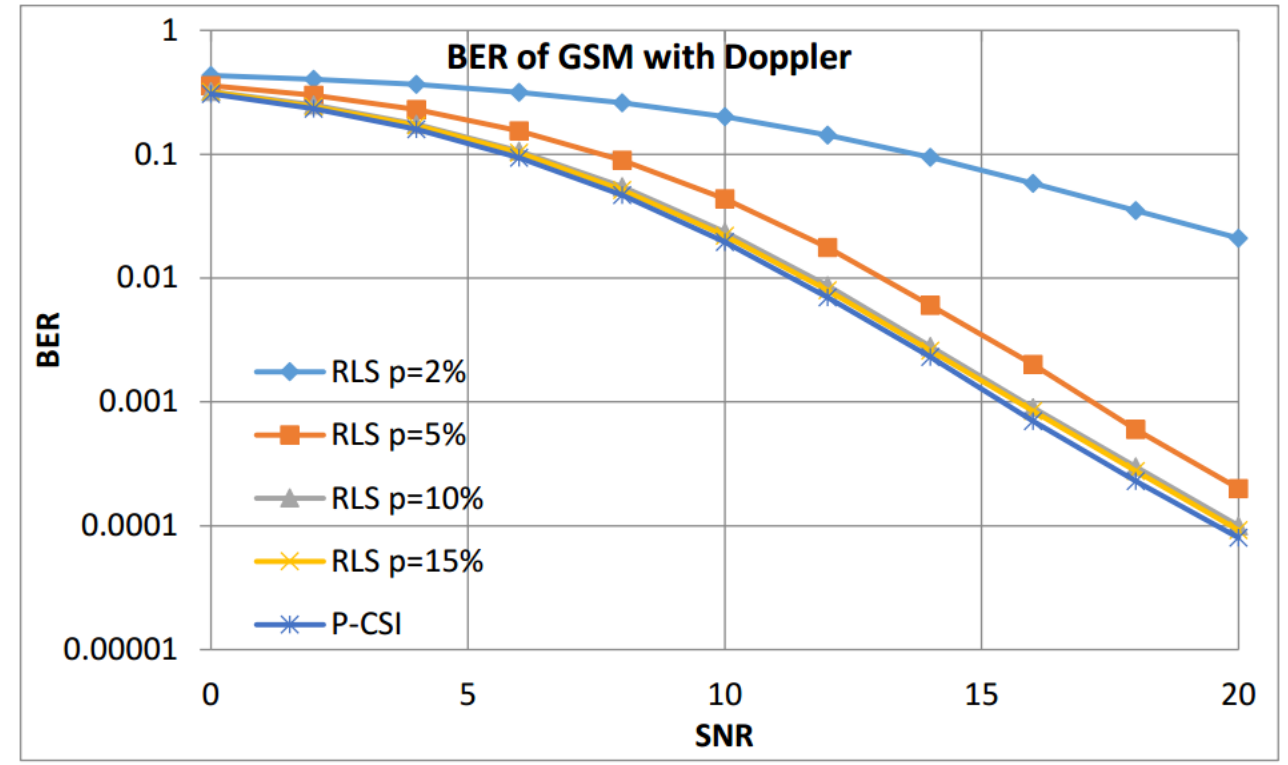

Figure 6. BER performance of the $4 \times 4$ underwater GSM-MIMO with Doppler-scaling factor.

\subsection{Fully Generalized Spatial Modulation}

The BER performance of the RLS channel estimator using a different number of pilots is compared with P-CSI for FGSM without Doppler-scaling factor over the time-varying MIMO channel provided in Figure 7. When the RLS channel estimator performance uses $2 \%$ of pilots as transmitted bits, the SNR gain difference is $9 \mathrm{~dB}$ between P-CSI and channel estimator at the target BER $10^{-2}$, and this performance difference is nearly the same for SM and GSM system, as seen in Figures 3 and 5. In comparison to P-CSI, the SNR gain difference is $1.85 \mathrm{~dB}$ using $5 \%$ of pilots used to estimate the time-varying channel at BER $10^{-2}$; this is far different than between P-CSI and the estimated results. Meanwhile using pilots $=$ numbers $10 \%$ and $15 \%$, the SNR gain difference at BER $10^{-2}$ is $0.32 \mathrm{~dB}$ and $0.21 \mathrm{~dB}$ in comparison of P-CSI. The RLS channel estimator BER performance using $10 \%$ and $15 \%$ pilots is greater than $2 \%$ and $5 \%$ number of pilots.

The BER performance of the RLS channel estimation method using pilots compared with a P-CSI for FGSM over time-varying MIMO channel in the presence of normalized Doppler-scaling factor is shown in Figure 8. As compared to a high number of pilots, the BER performance of $2 \%$ is not reasonable for SM, GSM, and FGSM with and without Doppler-scaling factor, as seen in Figures 3-8, respectively. At BER $10^{-2}$, the SNR difference gain as compared with P-CSI is $1.93 \mathrm{~dB}$, $0.34 \mathrm{~dB}$, and $0.25 \mathrm{~dB}$ while using $5 \%, 10 \%$, and $15 \%$ of pilots for estimating the channel. RLS channel estimator with and without Doppler-scaling factor in SM provides a better performance gain than GSM and FGSM, as depicted in Figures 3-8, respectively. This is because only one antenna is active in SM to transmit the data, and it helps the system to avoid inter-antenna interference as well as ICI. Additionally, the BER performance curve using with Doppler-scaling factor is shifted up approximately $10^{1}$ times than without Doppler-scaling factor. SM, GSM, and FGSM exhibit the same performance loss by adding and ignoring the Doppler-scaling factor. Moreover, the BER performance using the RLS channel estimator for $2 \%$ pilots is extremely low as compared to a high number of pilots in all SM, GSM, and FGSM. All the simulation results and analysis explicitly indicate that the RLS estimator is an appropriate channel estimation technique for the UWA-SMTs communication system. 


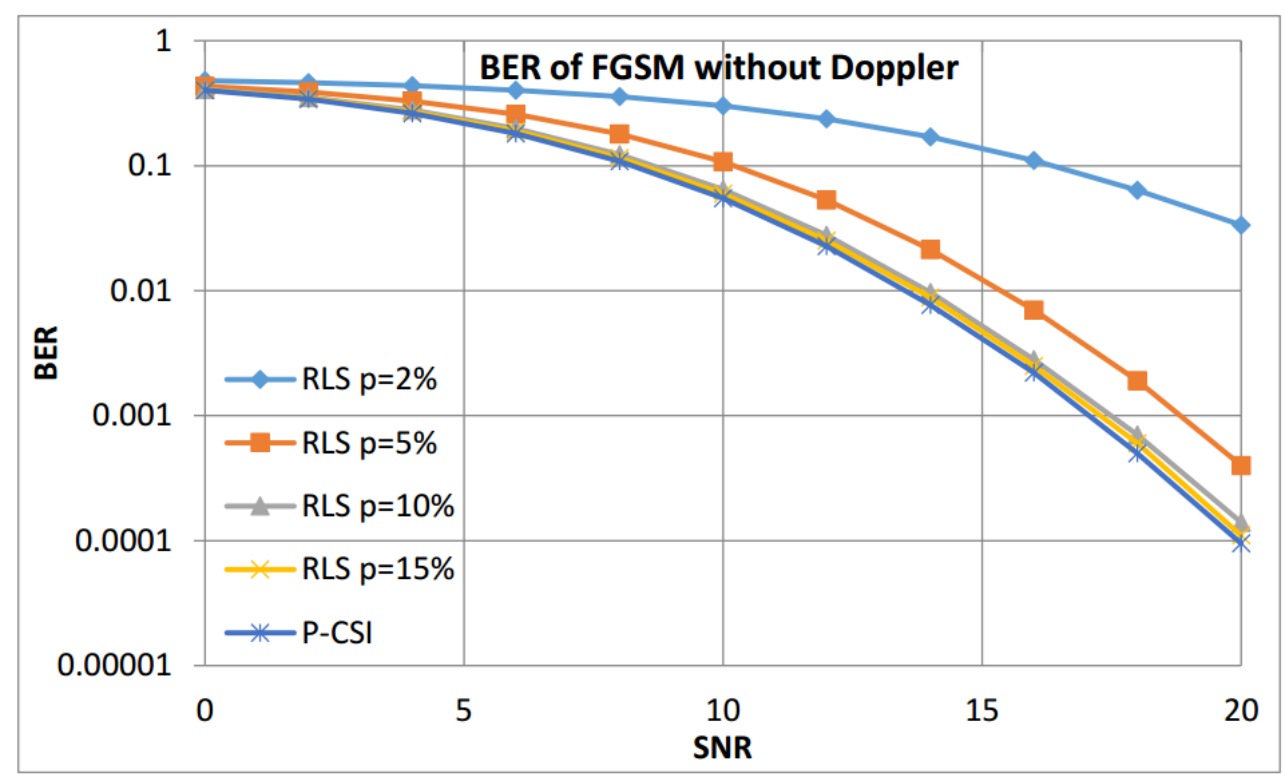

Figure 7. BER performance of the $4 \times 4$ underwater FGSM-MIMO without Doppler-scaling factor.

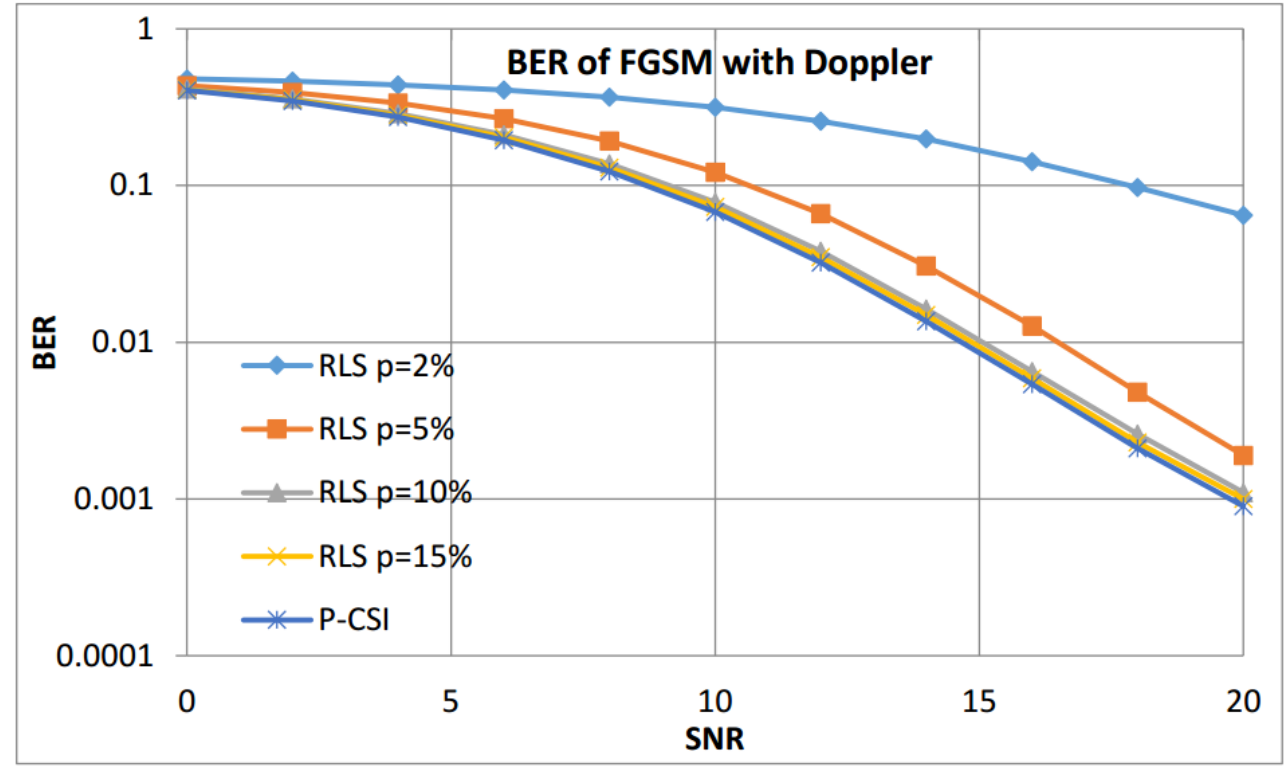

Figure 8. BER performance of the $4 \times 4$ underwater FGSM-MIMO with Doppler-scaling factor.

\subsection{Spectral Efficiency}

As mentioned above, the RLS can estimate the UWA-MIMO channel correctly, but the channel estimation is affected by the SMT spectral efficiency. The spectral efficiency of the UWA-SMT and the BER performance is a tread-off problem, where increasing the pilots improves the BER performance, but unfortunately, it reduces the spectral efficiency. The spectral efficiency of the SMTs compared with the pilot length is shown in Table 5. As the pilot length increases, the performance of RLS algorithms increases in terms of BER and MSE, but spectral efficiency is decreased. However, the total spectral efficiency of UWA-SMTs is higher than the conventional MIMO and such a pilot-length problem also exists in the conventional UWA-MIMO. In future work, we plan to improve the RLS to be used with a short pilot length in the UWA-SMTs and try different channel estimation techniques using a short pilot length to improve the UWA-SMT spectral efficiency. 
Table 5. Spectral Efficiency, MSE and BER Compared with the Pilot Length.

\begin{tabular}{llllll}
\hline $\begin{array}{l}\text { Number } \\
\text { of Pilots }\end{array}$ & $\begin{array}{l}\text { Used } \\
\text { Spectral } \\
\text { Efficiency }\end{array}$ & MSE & $\begin{array}{l}\text { BER FGSM } \\
(7 \text { pbcu) }\end{array}$ & $\begin{array}{l}\text { BER GSM } \\
\text { (6 pbcu) }\end{array}$ & $\begin{array}{l}\text { BER SM } \\
\text { (6 pbcu) }\end{array}$ \\
\hline $2 \%$ & $98 \%$ & 0.5678 & 0.3155 & 0.2003 & 0.2939 \\
$5 \%$ & $95 \%$ & 0.0953 & 0.1219 & 0.0436 & 0.1025 \\
$10 \%$ & $90 \%$ & 0.0180 & 0.0781 & 0.0235 & 0.0757 \\
$15 \%$ & $85 \%$ & 0.0091 & 0.0730 & 0.0220 & 0.0729 \\
$20 \%$ & $80 \%$ & 0.0060 & 0.0679 & 0.0195 & 0.0697 \\
\hline
\end{tabular}

\section{Conclusions}

SMTs are playing vital role in underwater sensor nodes because that can enhance battery life. SMTs need a semi-perfect channel known at the receiver side to be capable of estimating the constellation and index of information bits. In this paper, an adaptive pilot-based RLS algorithm is proposed to estimate the UWA channel for UWA-SMTs. The SMT data has been modulated by 16-QAM, and transmitted over $4 \times 4$ UWA-MIMO channel, under different pilot lengths $2 \%, 5 \%$, $10 \%$, and $15 \%$, at 7 bpcu for FGSM and 6 bpcu for SM and GSM. Using pilot-based RLS channel estimation, both the data and antenna indices are detected by ML optimal detector. The simulation results are conducted under a UWA-MIMO channel with and without Doppler-scaling factor. Based on the simulation results, we demonstrated that the pilot-based RLS channel estimator is capable for the SMTs-UWA-MIMO system and provide BER performance nearly as good as a P-CSI.

Author Contributions: The authors contributed equally to this manuscript.

Funding: This research was funded by The National Natural Science Foundation of China (61671394). This work is also supported by the National Key research and development Program of China (2018YFC0809200), Science and Technology Program of Shenzhen, China (JSGG20170414090428464), the Fundamental Research Funds for the Central Universities (20720170044).

Acknowledgments: The National Natural Science Foundation of China (61671394) supports this work. This work is also supported by the National Key research and development Program of China (2018YFC0809200), Science and Technology Program of Shenzhen, China (JSGG20170414090428464), the Fundamental Research Funds for the Central Universities (20720170044).

Conflicts of Interest: The authors declare no conflict of interest.

\section{References}

1. Ma, X.; Yang, F.; Liu, S.; Song, J. Channel estimation for wide-band underwater visible light communication: A compressive sensing perspective. Opt. Express 2018, 26, 311-321. [CrossRef]

2. Junejo, N.U.R.; Esmaiel, H.; Zhou, M.; Sun, H.; Qi, J.; Wang, J. Sparse Channel Estimation of Underwater TDS-OFDM System Using Look-Ahead Backtracking Orthogonal Matching Pursuit. IEEE Access 2018, 6, 74389-74399. [CrossRef]

3. Mostafa, M.; Esmaiel, H.; Mohamed, E. M. A comparative study on underwater communications for enabling C/U plane splitting based hybrid UWSNs. In Proceedings of the 2018 IEEE Wireless Communications and Networking Conference (WCNC), Barcelona, Spain, 15-18 April 2018; pp. 1-6.

4. Junejo, N.U.R.; Yan, J.; Adnan, S.; Chen, H.; Sun, H. Channel Estimation Using Pilot Method for Underwater Filter Bank Multicarrier System. In International Conference in Communications Signal Processing, and Systems; Springer: Singapore, 2017; pp. 1001-1009.

5. Shiu, D.S.; Foschini, G.J.; Gans, M.J.; Kahn, J.M. Fading correlation and its effect on the capacity of multielement antenna systems. IEEE Trans. Commun. 2000, 48, 502-513. [CrossRef]

6. Loyka, S.; Tsoulos, G. Estimating MIMO system performance using the correlation matrix approach. IEEE Commun. Lett. 2002, 6, 19-21. [CrossRef]

7. Chiani, M.; Win, M.Z.; Zanella, A. On the capacity of spatially correlated MIMORayleigh-fading channels. IEEE Trans. Inf. Theory 2003, 49, 2363-2371. [CrossRef] 
8. Catreux, S.; Driessen, P.F.; Greenstein, L.J. Simulation results for an interference-limited multiple-input multiple-output cellular system. IEEE Commun. Lett. 2000, 4, 334-336. [CrossRef]

9. Li, B.; Huang, J.; Zhou, S.; Ball, K.; Stojanovic, M.; Freitag, L.; Willett, P. MIMO-OFDM for High-Rate Underwater Acoustic Communications. IEEE J. Ocean. Eng. 2009, 34, 634-644.

10. Cheng, C.C.; Sari, H.; Sezginer, S.; Su, Y.T. New Signal Design for Enhanced Spatial Modulation with Multiple Constellations. In Proceedings of the 2015 IEEE 26th Annual International Symposium on Personal, Indoor, and Mobile Radio Communications (PIMRC), Hong Kong, China, 30 August-2 September 2015; pp. 872-876.

11. Renzo, M.D.; Haas, H.; Ghrayeb, A.; Sugiura, S.; Hanzo, L. Spatial Modulation for Generalized MIMO chalenges Oppotunities and Implementation. Proc. IEEE 2014, 102, 56-103. [CrossRef]

12. Mesleh, R.; Haas, H.; Ahn, C.W.; Yun, S. Spatial modulation a new low complexity spectral efficiency enhancing technique. In Proceedings of the 2006 First International Conference on Communications and Networking in China, Beijing, China, 25-27 October 2006; pp. 1-5.

13. Basar, E.; Aygolu, U.; Panayirci, E.; Poor, H.V. New trellis code design for spatial modulation. IEEE Trans. Wirel. Commun. 2011, 10, 2670-2680. [CrossRef]

14. Başar, E.; Aygölü, Ü.; Panayırc1, E.; Poor, H.V. Orthogonal frequency division multiplexing with index modulation. IEEE Trans. Signal Process. 2013, 61, 5536-5549. [CrossRef]

15. Hussein, H.S.; Esmaiel, H.; Jiang, D. Fully generalised spatial modulation technique for underwater communication. Electron. Lett. 2018, 54, 907-909. [CrossRef]

16. Hussein, H.S.; Elsayed, M.; Mohamed, U.S.; Esmaiel, H.; Mohamed, E.M. Spectral efficient spatial modulation techniques. IEEE Access 2018, 7, 1454-1469. [CrossRef]

17. Cheng, C.C.; Sari, H.; Sezginer, S.; Su, Y.T. New signal designs for enhanced spatial modulation. IEEE Trans. Wirel. Commun. 2016, 15, 7766-7777. [CrossRef]

18. Mesleh, R.Y.; Haas, H.; Sinanovic, S.; Ahn, C.W.; Yun, S. Spatial modulation. IEEE Trans. Veh. Technol. 2008, 57, 2228-2241. [CrossRef]

19. Di Renzo, M.; Haas, H.; Grant, P. Spatial Modulation for Multiple Antenna Wireless Systems: A Survey. IEEE Commun. Mag. 2011, 49, 182-191. [CrossRef]

20. Mohammadi, A.; Ghannouchi, F.M. Single RF front-end MIMO transceivers. In RF Transceiver Design for MIMO Wireless Communications; Springer: Berlin/Heidelberg, Germany, 2012; pp. 265-288.

21. Younis, A.; Serafimovski, N.; Mesleh, R.; Haas, H. Generalised spatial modulation. In Proceedings of the 2010 Conference Record of the Forty Fourth Asilomar Conference on Signals, Systems and Computers (ASILOMAR), Pacific Grove, CA, USA, 7-10 November 2010; pp. 1498-1502.

22. Narasimhan, T.L.; Raviteja, P.; Chockalingam, A. Generalized spatial modulation in large-scale multiuser MIMO systems. IEEE Trans. Wirel. Commun. 2015, 14, 3764-3779. [CrossRef]

23. Mesleh, R.; Ikki, S.S.; Aggoune, H.M. Quadrature spatial modulation. IEEE Trans. Veh. Technol. 2014, 64, 2738-2742. [CrossRef]

24. Jeganathan, J.; Ghrayeb, A.; Szczecinski, L. Spatial modulation: optimal detection and performance analysis. IEEE Commun. Lett. 2008, 12, 545-547. [CrossRef]

25. Basar, E.; Aygolu, U.; Panayirci, E.; Poor, H.V. Space-time block coded spatial modulation. IEEE Trans. Commun. 2010, 59, 823-832. [CrossRef]

26. Basar, E.; Aygolu, U.; Panayirci, E.; Poor, H.V. Performance of spatial modulation in the presence of channel estimation errors. IEEE Commun. Lett. 2011, 16, 176-179. [CrossRef]

27. Sugiura, S.; Hanzo, L. Effects of channel estimation on spatial modulation. IEEE Signal Process. Lett. 2012, 19, 805-808. [CrossRef]

28. Cavers, J.K. An analysis of pilot symbol assisted modulation for Rayleigh fading channels (mobile radio). IEEE Trans. Veh. Technol. 1991, 40, 686-693. [CrossRef]

29. Faiz, M.M.U.; Al-Ghadhban, S.; Zerguine, A. Recursive least-squares adaptive channel estimation for spatial modulation systems. In Proceedings of the 2009 IEEE 9th Malaysia International Conference on Communications (MICC), Kuala Lumpur, Malaysia, 14-17 December 2009; pp. 785-788.

30. Berger, C.R.; Zhou, S.; Preisig, J.C.; Willett, P. Sparse channel estimation for multicarrier underwater acoustic communication: From subspace methods to compressed sensing. IEEE Trans. Signal Process. 2010, 58, 1708-1721. [CrossRef] 
31. Esmaiel, H.; Jiang, D. Spectrum and energy efficient OFDM multicarrier modulation for an underwater acoustic channel. Wirel. Pers. Commun. 2017, 96, 1577-1593. [CrossRef]

32. Jeganathan, J.; Ghrayeb, A.; Szczecinski, L.; Ceron, A. Space shift keying modulation for MIMO channels. IEEE Trans. Wirel. Commun. 2009, 8, 3692-3703. [CrossRef]

33. Di Renzo, M.; Haas, H. Space shift keying (SSK) modulation with partial channel state information: Optimal detector and performance analysis over fading channels. IEEE Trans. Commun. 2010, 58, 3196-3210. [CrossRef]

34. Di Renzo, M.; Haas, H. Space shift keying (SSK-) MIMO over correlated Rician fading channels: Performance analysis and a new method for transmit-diversity. IEEE Trans. Commun. 2010, 59, 116-129. [CrossRef]

35. Jeganathan, J.; Ghrayeb, A.; Szczecinski, L. Generalized space shift keying modulation for MIMO channels. In Proceedings of the 2008 IEEE 19th International Symposium on Personal, Indoor and Mobile Radio Communications (PIMRC), Cannes, France, 15-18 September 2008; pp. 1-5.

36. Ntontin, K.; Di Renzo, M.; Perez-Neira, A.; Verikoukis, C. Adaptive generalized space shift keying. EURASIP J. Wirel. Commun. Netw. 2013, 2013, 43. [CrossRef]

37. Younis, A.; Basnayaka, D.A.; Haas, H. Performance analysis for generalised spatial modulation. In Proceedings of the 20th European Wireless Conference, Castelldefels, Barcelona, 14-16 May 2014; pp. 1-6.

38. Datta, T.; Chockalingam, A. On generalized spatial modulation. In Proceedings of the 2013 IEEE Wireless Communications and Networking Conference (WCNC), Shanghai, China, 7-10 April 2013; pp. 2716-2721.

39. Qasem, Z.A.; Esmaiel, H.; Sun, H.; Wang, J.; Miao, Y.; Anwar, S. Enhanced Fully Generalized Spatial Modulation for the Internet of Underwater Things. Sensors 2019, 19, 1519. [CrossRef]

40. Liu, Z.; Yang, T.C. On overhead reduction in time-reversed OFDM underwater acoustic communications. IEEE J. Ocean. Eng. 2013, 39, 788-800. [CrossRef]

41. Mason, S.F.; Berger, C.R.; Zhou, S.; Willett, P. Detection, synchronization, and Doppler scale estimation with multicarrier waveforms in underwater acoustic communication. IEEE J. Sel. Areas Commun. 2008, 26, 1638-1649. [CrossRef]

42. Sayed, A.H. Adaptive Filtering, 2nd ed.; Prentice-Hall: Englewood Cliffs, NJ, USA, 1991.

43. Sayed, A.H. Fundamentals of Adaptive Filtering; Wiley: Hoboken, NJ, USA, 2003.

(C) 2019 by the authors. Licensee MDPI, Basel, Switzerland. This article is an open access article distributed under the terms and conditions of the Creative Commons Attribution (CC BY) license (http:/ / creativecommons.org/licenses/by/4.0/). 